\title{
Comment
}

Neuroepidemiology 2009;33:266-267

DOI: $10.1159 / 000229782$

\section{Prediction of Hemorrhagic Transformation in Ischemic Stroke}

Akiyuki Hiraga

Department of Neurology, Chiba Rosai Hospital, Chiba, Japan

Hemorrhagic transformation (HT) of ischemic stroke (IS) occurs relatively frequently, especially in cardiac embolism. HT is a complex phenomenon, and mainly due to blood-brain barrier disruptions. Reperfusion injury and oxidative stress, leukocyte infiltration, vascular activation and dysregulated extracellular proteolysis are all potential HT triggers [1]. HT can occur spontaneously in IS, but is now a complication of widely used antithrombotic, anticoagulant or thrombolytic treatments for IS. The clinical spectrum of HT varies. While the majority of HT is not associated with the worsening of neurologic symptoms, symptomatic HT in the ischemic area worsens outcomes [2]. Thus, it is important to assess potential risk factors for HT. Baseline stroke severity, older age, large infarcts, time to reperfusion, thrombolytic treatment, white matter disease burden, and use of aspirin or anticoagulants are all associated with HT [2-4].

Terruso et al. [5] reported the overall frequency and risk factors for HT in a series of 240 IS patients studied between 2004 and 2006. Using the results from a retrospective hospital-based population study, the frequency of HT was $12 \%$. By multivariate analysis, HT was consistently associated with the sizes of ischemic areas as per previous reports. As all of the patients included in this study did not receive thrombolytic therapy, this study focused only on HT frequency for patients without thrombolytic treatment. Although there are many studies on the frequency and risk factors for HT in IS, most recent studies have focused on HT after thrombolytic treatment. The baseline information on HT frequency is very important in categorizing risk factors for HT in IS because thrombolytic treatments are becoming more widely used [3]. In this regard, the study of Terruso et al. [5] is important for our understanding of the natural history of HT in IS without thrombolytic treatments.

The reported frequency of HT in IS has varied widely. The frequency of HT due to cardiogenic embolism in the study of Terruso et al. [5] (18.9\%) was relatively lower than in previous studies.
One possible explanation for this low HT frequency is the timing of CT scans. In cardiogenic embolism, HT may occur anytime between the first few days to several weeks later. Variations of HT frequency in IS may also be due to timing, case mix, type and generation of the scanning modality used (e.g. MRI vs. CT), use of antithrombotic treatment, and several other factors [3]. In addition, differences in stroke frequency between countries should be considered as a reason for this variation. In fact, a recent prospective study in 4 centers in Italy showed that only $8.7 \%$ had early HT in all cases of IS, which is relatively lower than a previous study [6]. The incidence and frequency of stroke subtypes differ between countries, which may be due to differences in genetic factors, lifestyles or environmental factors. Therefore, studying the frequency and risk factors for HT in each country is necessary for understanding the likely impact on the prediction and treatment strategies for HT. Moreover, because changes in the frequency and type of stroke may occur in response to environmental or lifestyle changes in various countries and regions, HT frequency may vary from older reports. Since we can compare recent results with older studies, the study of Terruso et al. [5] may improve our understanding of HT. In order to provide comparisons with this study, future investigations focused on HT in IS are needed in many countries and regions.

This study focused on HT in anterior IS, but provided no information on posterior IS (e.g. cerebellar infarctions). Because numerous neurovascular differences exist between the anterior and posterior circulations, risk factors for HT in posterior IS remain unidentified. In fact, some reports from the 1990s suggested that cardiogenic embolism had a higher incidence than atherothrombosis in cerebellar infarctions $[7,8]$. HT in cerebellar infarction often increases disease severity, and may increase mortality risk (mainly due to brainstem compression). Thus, HT may impact both anterior and posterior IS. In the future, I hope that the authors will investigate the frequency of HT in posterior IS and its predictors.

Because HT may be related to reperfusion injury, the increased use of thrombolytic treatments in recent years could lead to an increasing incidence of this serious problem. The identification of predictive risk factors for HT in pretreatment stages will further aid the selection not only of thrombolytic, but also anticoagulant or antithrombotic treatments. It is important to understand the underlying mechanisms and identify early predictors for HT. Effective HT prediction is likely to have an effect on improving IS management strategies and outcomes.

\section{KARGER}

Fax +41613061234 E-Mail karger@karger.ch www.karger.com (c) 2009 S. Karger AG, Base

0251-5350/09/0333-0266\$26.00/0 


\section{References}

1 Wang X, Lo EH: Triggers and mediators of hemorrhagic transformation in cerebral ischemia. Mol Neurobiol 2003;28:229-244.

-2 Kerenyi L, Kardos L, Szász J, Szatmári S, Bereczki D, Hegedüs K, Csiba L: Factors influencing hemorrhagic transformation in ischemic stroke: a clinicopathological comparison. Eur J Neurol 2006;13:1251-1255.

- 3 Lindley RI, Wardlaw JM, Sandercock PAG, Rimdusid P, Lewis SC, Signorini DF, Ricci S: Frequency and risk factors for spontaneous hemorrhagic transformation of cerebral infarction. J Stroke Cerebrovasc Dis 2004;13:235-246

4 Aviv RI, d'Esterre CD, Murphy BD, Hopyan JJ, Buck B, Mallia G, Li V, Zhang L, Symons SP, Lee TY: Hemorrhagic transformation of ischemic stroke: prediction with CT perfusion. Radiology 2009;250:867-877.

5 Terruso V, D’Amelio M, Di Benedetto N, Lupo I, Saia V, Famoso G, Mazzola MA, Aridon P, Sarno C, Ragonese P, Savettieri G: Frequency and determinants for hemorrhagic transformation of cerebral infarction. Neuroepidemiology 2009;33:261-265.
6 Paciaroni M, Agnelli G, Corea F, Ageno W, Alberti A, Lanari A, Caso V, Micheli S, Bertolani L, Venti M, Palmerini F, Biagini S, Comi G, Previdi P, Silvestrelli G: Early hemorrhagic transformation of brain infarction: rate, predictive factors, and influence on clinical outcome: results of a prospective multicenter study. Stroke 2008;39:2249-2256.

7 Amarenco P, Hauw JJ, Gautier JC: Arterial pathology in cerebellar infarction. Stroke 1990;21:1299-1305.

8 Kase CS, Norrving B, Levine SR, Babikian VL, Chodosh EH, Wolf PA, Welch KMA: Cerebellar infarction: clinical and anatomic observations in 66 cases. Stroke 1993;24:76-83.

Akiyuki Hiraga, MD

Department of Neurology, Chiba Rosai Hospital

2-16 Tatsumidai-Higashi, Ichihara-shi

Chiba 290-0003 (Japan)

Tel. +81 43674 1111, Fax +81 43674 1151, E-Mail hiragaa@yahoo.co.jp 